\title{
Prevalence and risk factors for inappropriate birth weight for gestational age
}

\author{
Dittakarn Boriboonhirunsarn \\ Department of Obstetrics and Gynecology, Faculty of Medicine Siriraj Hospital, Mahidol University, \\ Bangkok 10700, Thailand
}

\begin{abstract}
Background: Infants with an inappropriate birth weight for their gestational age are more likely to develop complications during pregnancy and postpartum, and have increased long-term health risks.

Objectives: To determine the prevalence and risk factors for infants with inappropriate birth weight for their gestational age.

Methods: We enrolled 820 women with uncomplicated, singleton pregnancies who gave birth to a live born infant at term. Prepregnancy baseline and obstetric information were extracted from medical records, including body mass index (BMI), gestational weight gain, and infant birth weight. Prevalence of small-for-gestational age (SGA) and large-for-gestational age (LGA) infants was determined. We compared variables between groups to identify associated factors.

Results: Prevalence of SGA was 2.6\% and LGA was 10.5\%. Prepregnancy BMI and gestational weight gain were significantly higher in the LGA than in the SGA group $(P=0.041$ and $<0.001$, respectively). The birth weight and gestational weight gain, but not the prepregnancy BMI, were significantly different $(P<0.001)$. Logistic regression analysis determined that inadequate gestational weight gain significantly increased the risk of SGA (adjusted OR 3.20, 95\%CI 1.06 to 9.64, $P=0.039$ ), and significantly reduced the risk of LGA (adjusted OR 0.43, 95\% CI 0.20 to $0.91, P=0.028$ ). Excessive gestational weight gain significantly increased the risk of LGA (adjusted OR 2.00, 95\% CI 1.21 to 3.30, $P=0.006$ ). There was no significant association with prepregnancy BMI. Conclusions: Controlling gestational weight gain may improve maternal and neonatal outcomes.
\end{abstract}

Keywords: Gestational weight gain, large for gestational age, prepregnancy BMI, small for gestational age

Delivery of infants with an appropriate birth weight for their gestational age is one of the ultimate goals in obstetric care. However, inappropriate fetal growth is still an important public health problem in Thailand. Inappropriate birth weight-for-gestational age can be classified into: small-for-gestational age (SGA), which refers to birth weight $<10^{\text {th }}$ percentile, and large-for-gestational age (LGA), which refers to birth weight $>90^{\text {th }}$ percentile in the same population [1].

Infants with an inappropriate birth weight for their gestational age are more likely to develop complications during antepartum, intrapartum, and postpartum periods, and have increased long-term health risks. While SGA is associated with a greater chance of birth asphyxia, hypothermia, and abnormal

Correspondence to: Dittakarn Boriboonhirunsarn, Department of Obstetrics and Gynaecology, Faculty of Medicine, Siriraj Hospital, Mahidol University, Bangkok 10700, Thailand. E-mail: dittakarn.bor@mahidol.ac.th neurological development, LGA is associated with a greater proportion of cesarean deliveries, postpartum hemorrhage, and birth injury [2-5]. Special care is usually needed for these infants in order to prevent and treat complications.

Many factors are associated with SGA, such as short stature, prepregnancy underweight, poor gestational weight gain, prior history of SGA, smoking, and cardiovascular-associated diseases [6-11]. By contrast, maternal diabetes, obesity, multipara, excessive gestational weight gain, and post-term pregnancy were associated with LGA [3, 6, 9, 11$13]$.

However, there are only limited studies regarding this issue in Thailand. We aimed to determine the prevalence of inappropriate birth weight for gestational age among women with uncomplicated pregnancies who gave birth to a live born infant at term at Siriraj Hospital, a leading university hospital in Thailand. In addition, associated risk factors were also examined. 


\section{Methods}

After approval from Siriraj Institutional Review Board (SIRB no. Si 381/2010), we included 820 women with uncomplicated, singleton pregnancies who gave birth to a live born infant at term at Siriraj Hospital in this study. Pregnant women with medical or obstetric complications (e.g., diabetes mellitus, chronic or pregnancy-induced hypertension), uncertain gestational age, and identified fetal anomalies were excluded. Gestational age was estimated from the date of the mother's most recent menstrual period before pregnancy or ultrasonographic examination during pregnancy, or both.

Prepregnancy baseline characteristics and obstetrics information were extracted from medical records, including maternal age, parity, gestational age at delivery, prepregnancy body mass index (BMI), gestational weight gain, route of delivery, and infant birth weight.

Following guidelines issued by the Institute of Medicine (IOM); prepregnancy BMI was used to classify pregnant women into underweight, normal weight, overweight, and obese, and gestational weight gain [14].

Data for gestational age and infant birth weight were classified into 3 groups, namely, appropriate-forgestational age (AGA), small-for-gestational age (SGA), and large-for-gestational age (LGA) according to standard references of the Department of Pediatrics, Faculty of Medicine Siriraj Hospital. Prevalence and 95\% confidence interval (CI) for each birth-weight group was estimated. We compared variables between groups to assess possible associated factors.

Statistics, including the mean, standard deviation, number, and percentage were used to describe various characteristics as appropriate. Analysis of variance (ANOVA) followed by post hoc tests, and chi-square tests were used to compare groups as appropriate. Logistic regression analysis was used to determine independent risk factors for SGA and LGA infants, using the AGA group as a reference. Adjusted odds ratios (OR) and their 95\% CI were estimated. $P<0.05$ was considered statistically significant.

Table 1. Prepregnancy baseline and obstetric characteristics of the study population $(\mathrm{N}=820)$

\begin{tabular}{ll}
\hline Characteristics & $\mathbf{n} \mathbf{( \% )}$ \\
\hline Mean age \pm SD (years) & $28.4 \pm 6.3$ \\
Parity & \\
$\quad 0$ & $429(52.3)$ \\
1 & $301(36.7)$ \\
$\quad \geq 2$ & $90(11.0)$ \\
Mean prepregnancy BMI \pm SD $\left(\mathrm{kg} / \mathrm{m}^{2}\right)$ & $21.6 \pm 4.0$ \\
Prepregnancy BMI category & \\
$\quad$ Normal weight & $518(63.2)$ \\
$\quad$ Underweight & $162(19.8)$ \\
$\quad$ Overweight & $108(13.2)$ \\
$\quad$ Obese & $32(3.9)$ \\
Mean gestational weight gain \pm SD $(\mathrm{kg})$ & $14.1 \pm 4.7$ \\
Gestational weight gain category & \\
$\quad$ Adequate & $357(43.5)$ \\
$\quad$ Inadequate & $219(26.7)$ \\
$\quad$ Excessive & $244(29.8)$ \\
Mean gestational age \pm SD (weeks) & $38.7 \pm 1.1$ \\
Mean birth weight \pm SD (g) & $3100.7 \pm 381.0$ \\
Route of delivery & \\
Vaginal delivery & \\
Instrument vaginal delivery & $419(51.1)$ \\
Cesarean delivery & $15(1.8)$ \\
Male infant & $386(47.1)$ \\
\hline
\end{tabular}


Table 2. Birth weight for gestational age group $(n=820)$

\begin{tabular}{llll}
\hline Birth weight for gestational age group & n & Prevalence (\%) & 95\% CI \\
\hline AGA & 713 & 86.9 & $84.5,89.1$ \\
SGA & 21 & 2.6 & $1.7,3.9$ \\
LGA & 86 & 10.5 & $8.6,12.8$ \\
\hline
\end{tabular}

Table 3. Factors associated with infant birth weight for gestational age

\begin{tabular}{|c|c|c|c|c|}
\hline Characteristics & $\begin{array}{l}\text { AGA } \\
(n=713)\end{array}$ & $\begin{array}{l}\text { SGA } \\
(n=21)\end{array}$ & $\begin{array}{l}\text { LGA } \\
(n=86)\end{array}$ & ${ }^{a} \boldsymbol{P}$ \\
\hline Age (years) & $28.3 \pm 6.4$ & $26.3 \pm 6.8$ & $29.7 \pm 5.3$ & 0.05 \\
\hline Parity & & & & 0.27 \\
\hline 0 & $378(53 \%)$ & $14(67 \%)$ & $37(43 \%)$ & \\
\hline 1 & $257(36 \%)$ & $5(24 \%)$ & $39(45 \%)$ & \\
\hline$\geq 2$ & $78(11 \%)$ & $2(10 \%)$ & $10(12 \%)$ & \\
\hline Prepregnancy BMI (kg/m²) & $21.5 \pm 3.9$ & $20.4 \pm 5.3$ & $\begin{array}{l}22.6 \pm 4.0^{*} \\
(P=0.04)\end{array}$ & 0.02 \\
\hline Prepregnancy BMI category & & & & 0.12 \\
\hline Normal weight & $442(62 \%)$ & $15(71 \%)$ & $61(71 \%)$ & \\
\hline Underweight & $151(21 \%)$ & $3(14 \%)$ & $8(9 \%)$ & \\
\hline Overweight & $94(13 \%)$ & $3(14 \%)$ & $11(13 \%)$ & \\
\hline Obese & $26(4 \%)$ & $0(0)$ & $6(7 \%)$ & \\
\hline Gestational weight gain (kg) & $13.8 \pm 4.6$ & $13.9 \pm 5.7$ & $\begin{array}{l}16.3 \pm 4.9 * \\
(P<0.001)\end{array}$ & $<0.001$ \\
\hline Gestational weight gain category & & & & $<0.001$ \\
\hline Adequate & $318(45 \%)$ & $5(24 \%)$ & $34(40 \%)$ & \\
\hline Inadequate & $200(28 \%)$ & $10(48 \%)$ & $9(11 \%)$ & \\
\hline Excessive & $195(27 \%)$ & $6(29 \%)$ & $43(50 \%)$ & \\
\hline Gestational age (weeks) & $38.7 \pm 1.1$ & $39.0 \pm 1.1$ & $38.6 \pm 0.9$ & 0.42 \\
\hline Male infant & $370(52 \%)$ & $11(52 \%)$ & $52(61 \%)$ & 0.32 \\
\hline
\end{tabular}

${ }^{\mathrm{a}}$ ANOVA for comparison of means, Chi square test for comparison of proportions

*Significantly different from AGA group by Bonferroni post hoc comparison

\section{Results}

Prepregnancy BMI and gestational weight gain were significantly different between birth-weight groups and post hoc comparison determined that they were significantly higher in the LGA group compared with the AGA group. When prepregnancy BMI and gestational weight gain were categorized, significant difference was found between birth-weight group and gestational weight gain category, but not the prepregnancy BMI. No mothers of SGA infants were obese and most had normal prepregnancy weight. However, they were more likely to gain weight inadequately than infants in the AGA and LGA groups. By contrast, while most mothers of LGA infants had normal prepregnancy weight, they were more likely to be obese compared with mothers of infants in the other 2 groups. They were also more likely to have excessive gestational weight gain than the mothers of infants in the other 2 groups. No significant differences were found between birth-weight groups and maternal age, gestational age, parity, or neonatal sex.

Logistic regression analyses were conducted to determine independent risk factors for SGA and LGA, adjusting for variables, including prepregnancy BMI, gestational weight gain, maternal age, parity, and gestational age at delivery. In the analysis, overweight and obese women were grouped together because of their small number. The results are shown in Table 4. 
Table 4. Crude and adjusted odds ratios* of factors associated with SGA and LGA

\begin{tabular}{lllll}
\hline Risk factors & Crude OR & Adjusted OR & $\mathbf{9 5 \% C I ~}$ & $\mathbf{P}$ \\
\hline $\begin{array}{l}\text { SGA } \\
\text { Prepregnancy BMI category }\end{array}$ & & & & \\
$\quad$ Normal weight & 1.0 & 1.0 & & \\
$\quad$ Underweight & 0.58 & 0.52 & $0.14,1.87$ & 0.31 \\
$\quad \begin{array}{l}\text { Overweight or Obese } \\
\text { Gestational weight gain category }\end{array}$ & 0.74 & 0.84 & $0.23,3.07$ & 0.80 \\
$\quad$ Adequate & 1.0 & 1.0 & & \\
$\quad$ Inadequate & 3.18 & 3.20 & $1.06,9.64$ & 0.04 \\
$\quad$ Excessive & 1.96 & 1.69 & $0.50,5.73$ & 0.40 \\
LGA & & & & \\
Pre-pregnancy BMI category & 1.0 & 1.0 & & \\
$\quad$ Normal weight & 0.38 & 0.49 & $0.23,1.07$ & 0.07 \\
$\quad$ Underweight & 1.03 & 0.82 & $0.45,1.49$ & 0.51 \\
$\quad$ Overweight or Obese & & & & \\
Gestational weight gain category & 1.0 & 1.0 & $0.20,0.91$ & 0.03 \\
$\quad$ Adequate & 0.42 & 0.43 & $1.21,3.30$ & 0.01 \\
$\quad$ Inadequate & 2.06 & 2.00 & & \\
$\quad$ Excessive & & & & \\
$\quad$
\end{tabular}

*Adjusted for prepregnancy BMI and gestational weight gain category, age, parity, gestational age at delivery

Inadequate gestational weight gain increased the risk of SGA significantly by 3.2 times. For LGA, inadequate gestational weight gain reduced the risk significantly by $57 \%$, but excessive gestational weight gain significantly doubled the risk. We found no significant association between SGA or LGA and prepregnancy BMI.

\section{Discussion}

In this study, the prevalence of SGA was only $2.6 \%$, which was relatively lower than previously reported [2, 5, 10, 13, 15-17]. On the other hand, prevalence of LGA was $10.5 \%$, was comparable to previous reports from Thailand [18] and Vietnam [15]. However, the rate was slightly lower than that reported from Western countries [4, 9, 13, 16, 17]. The reasons for such differences could result from differences in the study population, especially in terms of race, ethnicity, and nutritional status. Inclusion of women with only low-risk, uncomplicated term pregnancies in this study, and improvement in antenatal care at our institution, particularly with regard to nutritional counseling and support, might minimize the prevalence of SGA in this population.

Prepregnancy BMI and gestational weight gain have been shown associated with infant birth weight and other obstetric outcomes [6, 12]. Previous reports have demonstrated that underweight pregnant women and those with inadequate gestational weight gain according to IOM guidelines, are at increased risk for SGA [9, 10, 13, 17, 19]. In addition, some studies reported that gestational weight gain, greater than recommended, reduced the risk of SGA $[10,13,17]$.

Similar findings were observed in the current study. The risk of SGA was increased by 3.2 times in pregnant women with inadequate weight gain. However, gaining more weight than recommended did not show a significantly risk reduction for SGA. By contrast with previous reports [10, 13, 15, 19], significant association with prepregnancy BMI was not demonstrated.

Consistent association between LGA and gestational weight gain above IOM guidelines for women in all prepregnancy weight ranges has been reported $[6,9,13,17,19]$. This was also observed in this study where excessive gestational weight gain doubled the risk of LGA. In addition, we found that inadequate gestational weight gain reduced the risk by $57 \%$, which was consistent with previous studies $[13,17]$.

Gestational weight gain appears a more important determinant of birth weight and fetal growth than prepregnancy BMI. In a systematic review, an association between gestational weight gain and 
birth weight was observed despite the use of different standard references, and various methods for characterizing gestational weight gain (total, rate, or by trimester). However, the associations appeared to be stronger when the rate of weight gain was used, rather than total weight gain [6].

Prepregnancy BMI and gestational weight gain can be modified to alter intrauterine exposures that can influence infant birth weight and intrauterine growth. With regard to birth weight for gestational age, pregnant women who follow the guidelines are likely to have better outcomes. Therefore, it appears rational that preventive measures should be implemented before and during pregnancy. Caring physicians should pay attention to nutritional status and support throughout pregnancy to ensure optimal gestational weight gain and reduce the risk of inappropriate infant growth. There should be surveillance of intrauterine fetal growth for at-risk pregnant women, for early detection and appropriate management to avoid maternal and neonatal complications.

There are some limitations to be noted. The sample size using in this study might compromise subgroup comparison, especially for the SGA group, and because of the unexpected low prevalence, which compromised power to detect some differences, there might be increased risk of type II error. Including only women with low-risk pregnancies makes it difficult to compare findings with other studies, in addition to the lack of uniformity of various definitions of both exposures and outcomes, and varied effects of race and ethnicity on the prevalence of both SGA and LGA. Therefore, the results might not be generalized to all populations.

Nonetheless, our findings provide additional evidence for the influence of factors related to maternal nutrition on infant birth weight and growth. Further large prospective studies in different cultural and ethnic settings should be conducted to confirm results and determine significant risks, and to explore further their impact on fetal growth.

\section{Conclusions}

The findings of this study should be applied in antenatal care; in particular nutritional surveillance, support, and monitoring should be controlled to mitigate the risks of inappropriate fetal growth and its related complications.

\section{Conflict of interest statement}

We received no funding from any sources for this study and there are no conflicts of interest to report.

\section{References}

1. Cunningham FG, Leveno KJ, Bloom SL, Hauth JC, Rouse DJ, Spong CY. Williams Obstetrics. 23 ed. New York: McGraw-Hill; 2010.

2. Clausson B, Gardosi J, Francis A, Cnattingius S. Perinatal outcome in SGA births defined by customised versus population-based birthweight standards. BJOG. 2001; 108:830-4.

3. Stotland NE, Caughey AB, Breed EM, Escobar GJ. Risk factors and obstetric complications associated with macrosomia. Int J Gynaecol Obstet. 2004; 87: 220-6.

4. Henriksen T. The macrosomic fetus: a challenge in current obstetrics. Acta Obstet Gynecol Scand. 2008; 87:134-45.

5. McIntire DD, Bloom SL, Casey BM, Leveno KJ. Birth weight in relation to morbidity and mortality among newborn infants. N Engl J Med. 1999; 340:1234-8.

6. Siega-Riz AM, Viswanathan M, Moos M-K, Deierlein A, Mumford S, Knaack J, et al. A systematic review of outcomes of maternal weight gain according to the Institute of Medicine recommendations: birthweight, fetal growth, and postpartum weight retention. Am J Obstet Gynecol. 2009; 201:339.e331-4.

7. McCowan L, Horgan RP. Risk factors for small for gestational age infants. Best Pract Res Clin Obstet Gynaecol. 2009; 23:779-93.

8. Murakami M, Ohmichi M, Takahashi T, Shibata A, Fukao A, Morisaki N, et al. Prepregnancy body mass index as an important predictor of perinatal outcomes in Japanese. Arch Gynecol Obstet. 2005; 271:311-5.

9. Savitz DA, Stein CR, Siega-Riz AM, Herring AH. Gestational weight gain and birth outcome in relation to prepregnancy body mass index and ethnicity. Ann Epidemiol. 2011; 21:78-85.

10. Watanabe $\mathrm{H}$, Inoue $\mathrm{K}$, Doi $\mathrm{M}$, Matsumoto $\mathrm{M}$, Ogasawara K, Fukuoka H, et al. Risk factors for term small for gestational age infants in women with low prepregnancy body mass index. J Obstet Gynaecol Res. 2010; 36:506-12.

11. Deierlein AL, Siega-Riz AM, Adair LS, Herring AH. Effects of pre-pregnancy body mass index and gestational weight gain on infant anthropometric outcomes. J Pediatr. 2011; 158:221-6.

12. Driul L, Cacciaguerra G, Citossi A, Martina MD, Peressini L, Marchesoni D. Prepregnancy body 
mass index and adverse pregnancy outcomes. Arch Gynecol Obstet. 2008; 278:23-6.

13. Nohr EA, Vaeth M, Baker JL, Sorensen TIa, Olsen J, Rasmussen KM. Combined associations of prepregnancy body mass index and gestational weight gain with the outcome of pregnancy. Am J Clin Nutr. 2008; 87:1750-9.

14. Rasmussen KM, Yaktine AL, eds. Weight gain during pregnancy: reexamining the guidelines. Washington, DC: The National Academies Press; 2009.

15. Ota E, Haruna M, Suzuki M, Anh DD, Tho le H, Tam NT, et al. Maternal body mass index and gestational weight gain and their association with perinatal outcomes in Viet Nam. Bull World Health Organ. 2011; 89:127-36.

16. Oken E, Kleinman KP, Belfort MB, Hammitt JK, Gillman MW. Associations of gestational weight gain with short- and longer-term maternal and child health outcomes. Am J Epidemiol. 2009; 170:173-80.

17. Park S, Sappenfield W, Bish C, Salihu H, Goodman D, Bensyl D. Assessment of the Institute of Medicine Recommendations for Weight Gain During Pregnancy: Florida, 2004-2007. Matern Child Health J. 2011; 15: 289-301.

18. Luengmettakul J, Boriboonhirunsarn D, Sutantawibul A, Sunsaneevithayakul P. Incidence of large-forgestational age newborn: a comparison between pregnant women with abnormal and normal screening test for gestational diabetes. J Med Assoc Thai. 2007; 90:432-6.

19. Choi SK, Park IY, Shin JC. The effects of pre-pregnancy body mass index and gestational weight gain on perinatal outcomes in Korean women: a retrospective cohort study. Reprod Biol Endocrinol. 2011; 9:6. doi: 10.1186/1477-7827-9-6. 